\title{
ANÁLISIS Y PROPUESTAS PARA LA ACREDITACIÓN DE PEDAGOGÍAS EN CHILE
}

\section{Macarena Domínguez, Lorena Meckes ${ }^{1}$}

\author{
RESUMEN
}

Este ensayo analiza la acreditación de las pedagogías en Chile, examinando las características actuales del sistema -criterios de evaluación, exigencia, consecuencias, normas, procedimientos, financiamiento- comparándolas con algunos rasgos que caracterizan a la acreditación en países con políticas robustas de aseguramiento de la calidad de los docentes recién egresados y contrastándolas con las características que debiera tener un proceso de evaluación cuando es utilizado con propósitos de accountability o rendición de cuentas. Se concluye identificando las falencias que deben ser superadas para potenciar la acreditación como pieza clave de un sistema que asegure la calidad de los futuros docentes en nuestro país.

Palabras clave: pedagogía, acreditación, calidad, accountability

\section{ANALYSIS AND PROPOSALS FOR ACCREDITATION OF PEDAGOGIES IN CHILE}

\section{ABSTRACT}

This essay examines the accreditation of initial teacher training programs in Chile-evaluation criteria, requirements, consequences, standards, procedures and financial arrangements- and compares them to accreditation processes in countries with a strong quality assurance of their teaching force. Chilean accreditation of initial teacher training is also compared to features that should be present in any assessment used for purposes of accountability. The essay concludes with the identification of the main weaknesses we should overcome in order to increase the contribution of the accreditation process to the quality assurance of the teaching force in our country.

Keywords: initial teacher training, quality assurance, accreditation, accountability

1 Investigadoras Centro de Estudios de Políticas y Prácticas en Educación, CEPPE. Pontificia Universidad Católica de Chile, Santiago, Chile

Contacto: mdomingl@uc.cl 


\section{ANÁLISIS Y PROPUESTAS PARA LA ACREDITACIÓN DE PEDAGOGÍAS EN CHILE}

Diversas son las señales que han llevado a que la calidad de la preparación de los docentes en Chile haya sido puesta en tela de juicio. Por una parte, a partir de 2002 se ha verificado un aumento explosivo de la matrícula y de la oferta de programas de formación inicial docente (FID) acompañado de la disminución en su selectividad (Cox, Meckes, Bascopé, 2011). Respecto de su calidad, revisiones de expertos internacionales (OCDE, 2004) y nacionales (Comisión sobre Formación Inicial Docente, 2005; Consejo Asesor Presidencial, 2006; Panel de expertos para una educación de calidad, 2010) han enfatizado la distancia observada entre los requerimientos del sistema escolar y la formación ofrecida. Finalmente, los egresados chilenos han mostrado magros resultados en el estudio comparativo sobre conocimientos disciplinarios y pedagógicos para enseñar matemática (TEDS-M) quedando posicionados en último y penúltimo lugar según si se analiza la preparación de quienes enseñarán en el primero o segundo ciclo básico (Ávalos y Matus, 2010).

Por su parte, las políticas últimamente han buscado incidir en los hitos que la evidencia comparada (OCDE, 2005; Barber y Mourshed, 2008; Wang, et al., 2003) identifica como puntos estratégicos en el aseguramiento de la calidad $(\mathrm{QA})^{2}$ de la preparación del cuerpo docente de un país:

(i) reclutar a quienes estudian para ser profesor de entre los mejores alumnos postulantes

(ii) monitorear y acreditar la calidad de los programas de formación de profesores y

(iii) establecer requisitos para entrar al ejercicio profesional u obtener una certificación definitiva como profesor

2 En este contexto, se usará QA en referencia a las políticas y regulaciones de un país, que aseguran la calidad de los egresados de la formación inicial docente y no al Sistema de Aseguramiento de la Calidad establecido en Chile por la Ley 12.129. 
Ingvarson, Schwille, Rowley, Tatto, Senk y Peck (2011) analizan las políticas y regulaciones de la FID de los 16 países participantes en TEDS-M, clasificándolos de acuerdo a su robustez en estos tres mecanismos claves para el aseguramiento de la calidad de los futuros profesores, encontrando que mientras más débil es el sistema de aseguramiento, más bajos eran los resultados obtenidos por los egresados de la FID en las pruebas de conocimientos disciplinarios y pedagógicos. En este estudio, Chile quedó clasificado en el grupo de países más desregulado y con un sistema de QA más débil, junto con Omán, Tailandia, Georgia y Filipinas. En el otro extremo -con sistemas robustos y buenos resultados- se ubican China -Taipéi-, Canadá, y la Federación Rusa.

De acuerdo al estudio, países como Chile con escasa o nula regulación, se caracterizan por:

- Reclutamiento y selección: no presentan estándares mínimos que se deban alcanzar para ingresar a estudiar pedagogía (por ejemplo un puntaje que se deba superar en las pruebas de admisión) ni políticas para hacer de la profesión una carrera atractiva.

- Los procesos de acreditación son voluntarios u obligatorios, pero no existen atribuciones para cerrar los programas que no acreditan. La acreditación no se basa en estándares nacionales para los programas ni tampoco en estándares mínimos de resultados obtenidos por quienes se gradúan de ellos.

- Basta el título otorgado por la institución formadora para ejercer como profesor. No hay estándares para los graduados o si los hay, no se requiere aprobar una evaluación externa para poder acceder al ejercicio profesional. Tampoco existe un periodo de autorización provisoria con la correspondiente exigencia de aprobar una evaluación al término del periodo.

Aunque el estudio se realizó el 2008, y ha habido algunos cambios, es posible aseverar que las características que hicieron clasificar a Chile como un país débil en su QA de los futuros profesores, siguen vigentes. A excepción de las iniciativas para mejorar las condiciones salariales de los profesores iniciales con buen rendimiento (las que buscan generar un mayor atractivo hacia 
168 ANÁLISIS Y PROPUESTAS PARA LA ACREDITACIÓN DE PEDAGOGÍAS EN CHILE - M. Domínguez, L. Meckes

la profesión), las demás políticas recientemente implementadas, si bien se orientan en la dirección correcta, aún no representan un cambio que permita aseverar que Chile ha fortalecido su sistema de QA.

Sin duda, las nuevas iniciativas de política se han focalizado en los dos extremos del trayecto de formación -ingreso y egresosin prestar mayor atención a los restantes mecanismos de QA, particularmente a la acreditación de los programas de formación inicial docente, que de acuerdo a expertos nacionales, no da garantías de asegurar calidad (panel de expertos, 2010) y que será precisamente nuestro objeto de análisis.

\section{Características generales de la acreditación de carreras de pedagogía}

Desde que en 2006 entró en vigencia la Ley 20.129, la acreditación de carreras que conducen al título profesional de educador o profesor es obligatoria y prerrequisito para recibir financiamiento estatal, tanto para las instituciones como para los alumnos. Esta función es realizada por agencias privadas, autorizadas y supervisadas por la Comisión Nacional de Acreditación (CNA) ${ }^{3}$.

El proceso de acreditación de programas de pregrado define tres etapas a las que deben someterse las unidades académicas: (a) evaluación interna o autoevaluación; (b) evaluación externa realizada por una agencia acreditadora cuyo comité de pares debe analizar el informe de autoevaluación y visitar la unidad académica; y (c) pronunciamiento o juicio de acreditación realizado por la agencia sobre la base de los criterios de evaluación, del informe de autoevaluación de la carrera, y el informe de pares evaluadores. Como resultado es posible (1) no acreditar la carrera, (2) acreditar la carrera por un máximo de 7 años, y (3) acreditando la carrera, no acreditar alguna de sus sedes, modalidades o jornadas. Las sedes, jornadas o modalidades que son acreditadas, deben serlo

3 Para mayor detalle sobre la institucionalidad del sistema chileno de acreditación ver Lemaitre y Zapata (2003) y Zapata y Tejeda (2009). 
por el mismo número de años (Comisión Nacional de Acreditación, $2008)^{4}$.

\section{¿Qué características de la acreditación de las pedagogías deberían mejorar?}

En las siguientes secciones se pretende analizar ciertos rasgos de la acreditación de carreras de pedagogía con énfasis en aquellos que podrían mejorarse para asegurar mejores resultados en la preparación de sus egresados. Los criterios utilizados para este análisis son, por una parte, el contraste de nuestra acreditación con algunas de las características que la acreditación tiene en aquellos países cuyos sistemas de QA son "robustos", de acuerdo a Ingvarson, et al. (2011), y por otra, su comparación con algunas características de los sistemas de evaluación con propósitos de accountability o rendición de cuentas.

Los países con "sistemas robustos" de QA se caracterizan por estar a cargo de una agencia independiente con atribuciones para imponer fuertes consecuencias para los programas no acreditados (cierre de los programas o suspensión de su financiamiento), estar referidos a estándares de procesos que son específicos para las pedagogías y en algunos casos también a estándares de resultados que deben ser alcanzados por los graduados (Ingvarson et al., 2011). Al contrastar nuestra acreditación de las carreras de pedagogía con estos rasgos, surgen tres dimensiones en que sería posible mejorar para incrementar su contribución al QA de los futuros profesores: a) los criterios de evaluación de la acreditación b) la exigencia de estos, y c) las consecuencias asociadas a sus resultados.

4 La institucionalidad del sistema chileno de acreditación comparte algunas características con otros sistemas del mundo, por ejemplo la creación por ley de agencias de acreditación y la revisión de pares. Sin embargo, en la mayoría de los países, la agencia acreditadora es única y se caracteriza por ser una agencia de gobierno nacional (o estatal para aquellos países con gobiernos federales). Además, en algunos casos depende del Ministerio de Educación o de colegios profesionales, y debe ser acreditada. Y en cualquier caso, aun cuando se trata de agencias privadas independientes como NCATE en los EE.UU., se trata de agencias sin fines de lucro (Ingvarson et al., 2011). Más adelante se revisarán las repercusiones de estas diferencias para nuestro sistema de acreditación. 
170 ANÁLISIS Y PROPUESTAS PARA LA ACREDITACIÓN DE PEDAGOGÍAS EN CHILE - M. Domínguez, L. Meckes

Por otra parte, los procedimientos de evaluación con propósitos de rendición de cuentas o accountability, deben responder a exigencias distintas que las evaluaciones que tienen propósitos exclusivamente formativos o de mejoramiento continuo. Es posible clasificar las funciones que Zapata y Tejeda (2009) identifican como convergentes para los sistemas de acreditación en el plano internacional en dos grandes grupos: formativos (apoyo a la planificación institucional y del sistema, fomento y mejoramiento continuo de la calidad) y de accountability (información pública sobre calidad, reconocimiento y rankings, certificación de la calidad, control, direccionamiento del financiamiento). Si bien en Chile se ha enfatizado el propósito formativo de la acreditación, sin duda la publicación de sus resultados y su obligatoriedad en el caso de pedagogía y medicina, incorpora con fuerza el propósito de accountability. Sin perjuicio de los beneficios que el sistema chileno ha significado para el mejoramiento continuo de las instituciones (Scharager y Aravena, 2010) $)^{5}$, nuestro interés es examinar en qué medida su diseño es consistente con una evaluación orientada al accountability.

Las evaluaciones utilizadas para accountability requieren, entre otras cosas, asegurar su validez e imparcialidad, contar con un referente o marco (estándares de contenido y de desempeño de aquello que se está evaluando), incluir medidas diversas o muestras de distinta complejidad, "puntos de corte" que distingan niveles de desempeño, mecanismos para eliminar o disminuir los sesgos y errores de medición, mecanismos de control del cumplimiento de las condiciones estandarizadas de las evaluaciones (Fuhrman, 2003; Ravela, et al., 2008). Estas últimas exigencias necesitan de un sistema diseñado para garantizar la independencia y neutralidad del evaluador y la estandarización de sus procedimientos, instrumentos y criterios para asegurar la comparabilidad de los resultados.

Considerando lo anterior, es posible revisar en el proceso chileno: d) las normas y procedimientos, y e) el financiamiento de la acreditación.

5 Para mayor detalle sobre la relación entre acreditación y aseguramiento de la calidad de la formación docente y de las carreras de educación superior en el contexto internacional ver Wilson y Youngs (2006) y Stensaker (2007). En el contexto nacional ver Navarro (2008) y Scharager y Aravena (2010). 
a) Criterios de acreditación referidos a estándares, que incorporen los resultados de sus egresados y se enfoquen en procesos pedagógicos y curriculares específicos para pedagogía

Los sistemas de acreditación pueden concebir la calidad de un programa como la medida en que éste (1) logra los propósitos internamente establecidos (coherencia), (2) satisface las expectativas de grupos de interés relevantes, o el grado en que (3) alcanza estándares definidos externamente (Bills, et al., 2008).

De acuerdo a la CNA"las carreras que se incorporen al proceso de acreditación serán evaluadas respecto a: a) el perfil de egreso de la respectiva carrera, que deberá considerar el estado de desarrollo y actualización de los fundamentos científicos, disciplinarios o tecnológicos que subyacen a la formación que se propone entregar ${ }^{6}$ y las orientaciones fundamentales provenientes de la declaración de misión y propósitos, y fines institucionales; y b) el conjunto de recursos y procesos mínimos que permiten asegurar el cumplimiento del perfil de egreso definido para la carrera." (Comisión Nacional de Acreditación, 2008). De esta definición es posible inferir que en Chile la acreditación observa con especial énfasis la calidad entendida como consistencia interna.

En contraste, los sistemas que son más sólidos para asegurar la calidad de los egresados de pedagogía, se caracterizan por procesos de acreditación referidos a estándares, tanto de procesos y condiciones institucionales, como de resultados, es decir, de desempeño y conocimientos que deben demostrar quienes egresan de los programas (Ingvarson et al., 2006; Rothstein et al., 2009). Más aún, en los últimos años se ha observado un giro de los sistemas de acreditación desde un foco en insumos y procesos (requisitos para ingresar a la carrera, infraestructura, malla curricular, cuerpo docente, etc.) a otro depositado en estándares y resultados, que

6 No obstante esta definición, no existe en el proceso de acreditación chileno ningún contraste de la carrera o su perfil de egreso con referentes externos o con "el estado de desarrollo y actualización de los fundamentos científicos, disciplinarios o tecnológicos" de la profesión. El uso en la evaluación de los criterios propuestos por la CNAP para las carreras de educación podrían cumplir esta función; sin embargo, no está muy claro si la ley y normas del proceso se refieren a esto como consistencia externa. 
172 ANÁLISIS Y PROPUESTAS PARA LA ACREDITACIÓN DE PEDAGOGÍAS EN CHILE - M. Domínguez, L. Meckes

es el que hoy predomina en Europa (Euridyce, 2010; Ingvarson et al., 2011).

Los criterios chilenos de evaluación para pedagogía han sido definidos por el Comité Técnico de Educación de la Comisión Nacional de Acreditación de Pregrado (CNAP) antes de la aprobación de la ley, estableciendo tres dimensiones: (1) perfil de egreso y resultados, (2) condiciones mínimas de operación y (3) autorregulación. A pesar de lo que el título de la primera dimensión podría sugerir, los criterios no consideran los resultados del programa en términos de competencias demostradas. Solo consideran resultados en términos de tasas de retención, aprobación, titulación, etc.

Adicionalmente, los aspectos a los que la acreditación pone más atención no son aquellos relacionados con las dimensiones pedagógicas o curriculares. Por el contrario, tienen más presencia aspectos organizacionales que ya están considerados en la acreditación institucional, en las áreas de gestión institucional y docencia de pregrado. Una simple comparación de la ley y los documentos que orientan desde la CNA los procesos de acreditación ${ }^{7}$, lleva a concluir que existe una relativa superposición de los criterios de acuerdo a los cuales se evalúan instituciones y carreras. La definición de propósitos, la estructura organizacional, administrativa y financiera, las normativas, políticas y mecanismos administrativos asociados a recursos humanos, los mecanismos y procedimientos de diagnóstico, de recolección de información, de autoevaluación, son ejemplos de los ámbitos en que esta superposición se puede observar.

Sería lógico suponer, que si el sistema de acreditación institucional funciona apropiadamente ${ }^{8}$, los procesos organizacionales que se observan en la institución, debieran verificarse también en los programas de pregrado, y por ende, se esperaría que los criterios para acreditar carreras fueran más específicos. Para un sistema

7 Nos referimos aquí a los "Términos de referencia para las áreas contempladas en el proceso de acreditación institucional", "Normas y procedimientos acreditación institucional", "Normas y procedimientos acreditación de pregrado", "Criterios de evaluación de carreras de educación" y "Operacionalización criterios de evaluación en procesos de acreditación".

8 Y su cobertura llega al 100\% de las instituciones, pues de acuerdo a la CNA, al 2010 ya el 95\% de las universidades del país están en el sistema. 
de acreditación de programas obligatorio, se requeriría un mayor énfasis en aspectos propios del nivel de la unidad académica que ofrece la carrera, así como aspectos propios del programa y de sus resultados.

Se esperaría que en el futuro los criterios para carreras de pedagogía fueran actualizados y reconstruidos sobre una sólida base de consensos nacionales y sobre la evidencia empírica nacional e internacional en la materia, que considera: relación y coherencia entre la malla curricular y cada uno de sus cursos con los estándares o competencias de egreso esperados para los futuros profesores; secuencia apropiada de experiencias y cursos; solidez de la formación teórico disciplinaria (número o proporción de cursos de la disciplina que enseñará); profunda integración entre disciplina y didáctica específica de esta disciplina (incluida la evaluación de aprendizajes); profunda y temprana integración de experiencias prácticas progresivamente ligadas al ámbito escolar y a la labor docente; estructura de monitoreo, tutoría o mentoría, y seguimiento de las experiencias de práctica que muestren un fluida colaboración entre instituciones formadoras y centros de práctica; calificación del cuerpo docente que considere su vinculación con el mundo escolar y/o experiencia en aula; oportunidades de aprendizaje en torno a la investigación sobre la práctica pedagógica; coherencia de las formas de evaluación de los alumnos durante la formación y al egreso y titulación con las competencias que de acuerdo a los estándares nacionales se espera demuestren, entre otros Darling-Hammond y Bransford, 2005; Darling-Hammond, 2006; Levine, 2006; NCATE, 2008; National Research Council, 2010; Varas et al., 2008; Sotomayor et al., 2010; Quality Assurance Agency, QAA, 2007; Teacher Development Agency, TDA 2006 y 2008; Teacher Education Accreditation Council, TEAC, 2009.

Para que el sistema cumpla con su cometido de asegurar la calidad de los programas de pedagogía, se debiera incorporar a los procesos de acreditación la definición de estándares para profesores egresados de educación básica y media y orientaciones para la FID, que a partir de 2009 el MINEDUC ha estado construyendo. Asimismo, sería recomendable incluir entre los criterios de acreditación los 
resultados de la formación en términos de proporción de egresados que logra los estándares, según exámenes externos de egreso, los que debieran a su vez estar alineados a dichos estándares. Esta función podría cumplir la evaluación INICIA o el examen de excelencia profesional docente propuesto por el proyecto de ley que el ejecutivo envió al Congreso, aunque para ello sería necesario que este evalúe competencias pedagógicas además de conocimientos disciplinarios y establezca un claro deslinde para diferenciar entre quienes aprueban la evaluación final (y por lo tanto logran los estándares mínimos) y quiénes no.

El proyecto de ley que establece este examen de excelencia profesional docente mandata que deberá ser rendido por todo profesor que postule a ser contratado por primera vez en un establecimiento subvencionado y sus resultados -agregados por institución-deberán ser públicos. Sin embargo, el proyecto no contempla la definición de un puntaje de corte que distinga entre quienes aprueban o no la evaluación, ni tampoco que el examen deba evaluar más que conocimientos disciplinarios. Si estas omisiones no se modifican en el Congreso, solo será posible conocer el ranking de resultados de las instituciones, pero no qué proporción de sus egresados logra un nivel adecuado para comenzar su ejercicio profesional.

\section{b) Criterios de acreditación más exigentes}

Al examinar los criterios con que son evaluados los programas de FID, es posible constatar que para no acreditarse, estos deben presentar niveles de calidad extremadamente deficitarios. Efectivamente, al analizar las rúbricas contenidas en el documento "Operacionalización criterios de evaluación en procesos de acreditación" (CNA), las descripciones correspondientes al nivel "no acredita" reflejan extremas debilidades de los programas. Por ejemplo: "no presenta propósitos y fines institucionales"; "el perfil de egreso no está definido o no tiene validación interna o externa"; "existe inconsistencia entre perfil, currículo y estrategias pedagógicas"; "no existen indicadores de resultados, de progresión, egreso, titulación"; "condiciones de operación insuficientes para funcionamiento aceptable"; "RR.HH. insuficientes en número, calificaciones y dedicación”; etc. 
Dos problemas se hacen evidentes al revisar las características que tendrían los programas que no acreditan:

- Si las carreras que no se acreditan son tan deficientes, entonces resulta inverosímil que ello no tenga consecuencias significativas; y,

- Si no existen carreras así de deficientes, la categoría "no acredita" se hace inaplicable. Esto quiere decir que el "punto de corte" establecido por el documento está tan por debajo de la expectativa, que es improbable que una carrera resulte no acreditada.

Para evitar estas distorsiones, es recomendable que existan estándares específicos para los programas de FID (Panel de Expertos para una Educación de Calidad, 2010), o bien, mejores instrumentos para su evaluación que consideren niveles de desempeño del programa y características mínimas de su oferta que sean más exigentes y fruto de un acuerdo nacional.

c) Establecer consecuencias asociadas a los resultados de la acreditación

Otra característica de la acreditación en países con elevados niveles de aseguramiento de la calidad de sus futuros profesores (Ingvarson, et al., 2011) es asociar consecuencias severas a la no acreditación de las instituciones formadoras como el cierre de la carrera o la suspensión total de su financiamiento (que es primordialmente público).

Si la acreditación chilena solo tuviera fines formativos, sería comprensible que no se deriven consecuencias de no acreditar. Pero, la Ley 20.129 establece la obligatoriedad de la acreditación para las pedagogías, y pese a ello, para aquellos programas que no se presentan al proceso o que no resultan acreditados, no define más consecuencias que no poder "acceder a ningún tipo de recursos otorgados directamente por el Estado o que cuenten con su garantía, para el financiamiento de los estudios de sus nuevos alumnos" (Ley 20.129). Se desconoce cuánto pesan estas sanciones en un sistema de educación superior cuyo financiamiento es mayoritariamente privado 
176 ANÁLISIS Y PROPUESTAS PARA LA ACREDITACIÓN DE PEDAGOGÍAS EN CHILE - M. Domínguez, L. Meckes

(Zapata y Tejeda, 2009) -es decir, lo aportan las familias- ya que no toda carrera no acreditada termina por cerrar.

Para mejorar este aspecto, el panel de expertos (2010) propuso consecuencias significativas, como la imposibilidad de aceptar nuevos alumnos a aquellos programas que por segunda vez se acrediten por menos de cuatro años. Es necesario comentar que desde la perspectiva de los sistemas de evaluación, establecer un "punto de corte" en cuatro años equivaldría a elevar el nivel de exigencia y hacer desaparecer la distinción entre programas acreditados y no acreditados junto con las categorías de uno a tres años.

Al mismo tiempo que el sistema de acreditación crezca en validez y confiabilidad, se puede pensar en otro tipo de consecuencias, como las que se imponen en EE.UU., donde para presentarse al examen de habilitación para ejercer, el profesor debe tener un grado académico de un programa de formación acreditado, lo que es otro modo de motivar a dichos programas a elevar la calidad de la formación para acreditarse y ofrecer un futuro profesional a sus estudiantes.

d) Establecer normas y procedimientos estandarizados

Como se ha señalado anteriormente, los procesos de acreditación de carácter obligatorio cumplen con propósitos de accountability, además de dar retroalimentación para un mejoramiento continuo. Las exigencias de neutralidad, consistencia, confiabilidad y comparabilidad de los resultados para cualquier sistema de evaluación que, como este, produce resultados que son públicos y utilizados para rendición de cuentas, son mayores que para una evaluación más cercana a una asesoría externa con fines netamente formativos.

Se esperaría que la calidad de la formación ofrecida por dos programas que han acreditado por el mismo número de años sea si no equivalente, muy semejante, y superior al nivel que presenta un tercer programa acreditado por menos años. Para responder a esta razonable expectativa, es necesario que los procedimientos de evaluación incluyan procesos para asegurar la equivalencia de los criterios utilizados en cada caso, es decir, es necesario establecer mecanismos que permitan "estandarizar" la evaluación, para que 
los resultados no dependan de la agencia acreditadora, los pares evaluadores ni los procedimientos utilizados.

Al revisar la ley, las "normas y procedimientos" y los documentos para la acreditación de carreras de pregrado ${ }^{9}$, encontramos que:

- El procedimiento de convocatoria y contratación de pares evaluadores por parte de las agencias no está normado. A pesar de que se estipula que deben cumplir con los mismos requisitos que exige la CNA a sus pares, no es muy clara la relación entre unos y otros. La CNA tiene una nómina de pares evaluadores, aunque no todos los pares de las agencias aparecen en esta lista. Tampoco es claro si la participación de un par evaluador extranjero es un requisito uniforme.

- La capacitación de dichos pares evaluadores no es estandarizada y depende de cada agencia.

- La visita del comité de pares no está normada, en términos de su duración (se sugiere de tres días), meticulosidad, y exhaustividad con relación a visitar y revisar todas las sedes, jornadas, y modalidades.

- Respecto de la acreditación de programas que se imparten en diferentes modalidades, jornadas (diurno, vespertino, etc.) o sedes, no existen normas específicas. Se deduce de la definición conceptual que aparece en el manual y en las normas y procedimientos de la CNA que cada programa que solicita la acreditación debe evaluar todas sus versiones. Junto con no estar esto en la ley, carece de mayores especificaciones. Por ejemplo, no existe ninguna orientación a las agencias ni a los pares acerca de cómo establecer los años de acreditación de un programa en el caso que presente sedes o modalidades con niveles de calidad disímiles (¿Se otorga el número de años que correspondería al

9 Incluidos los documentos de la CNA citados anteriormente, y además el "Reglamento agencias; Resolución exenta 165-3", "Sistema de supervisión y seguimiento agencias", "Términos de referencia concurso público pares evaluadores", "Manual de pares evaluadores", "Guía para la evaluación externa con fines de acreditación carreras y programas de pregrado", "Manual para el desarrollo de procesos de autoevaluación. Carreras y programas de pregrado", "Criterios generales de evaluación para carreras profesionales", "Criterios de evaluación de carreras de educación", las "Guías de formularios" (A, B y C) y las circulares 1 a la 15 . 
programa con mayores debilidades? ¿O el número de años que correspondería al programa con mayores fortalezas? ¿Se calcula el promedio del número de años que se asignaría a cada sede?).

- Al revisar los acuerdos de acreditación se puede concluir que tampoco existen parámetros comunes para su redacción ${ }^{10}$.

Considerando las definiciones que quedan al arbitrio de las agencias acreditadoras y que por ende no son comunes a todos los procesos de acreditación, es difícil pensar que sus resultados sean comparables. Si bien la CNA tiene la función de supervisar a las distintas agencias, no existe evidencia disponible que permita observar el ejercicio de esta función. El 2010, la CNA entregó la memoria sobre su actividad desde 2007. No aparece en ella descripción alguna de los procesos implementados para supervisar a las agencias ni análisis del funcionamiento de ellas, estudiando, por ejemplo, la relación entre agencias e instituciones acreditadas, el promedio de años de acreditación que otorga cada una, los antecedentes de los pares evaluadores que efectivamente participan en los procesos, etc.

Finalmente, son las instituciones de FID las que seleccionan a la agencia acreditadora. Esta norma resulta coherente para un sistema voluntario orientado exclusivamente al mejoramiento continuo, pero no lo es en un sistema obligatorio usado también para accountability. Tal vez sea de utilidad hacer aquí una comparación con los procedimientos utilizados para la corrección de preguntas de desarrollo en los exámenes estandarizados. Además de una capacitación rigurosa para asegurar que se evalúa con los mismos criterios, las respuestas son asignadas aleatoriamente a quien corrige y el examinado no puede elegir quién revisará su respuesta. De este modo, se busca generar condiciones de neutralidad de la evaluación y de comparabilidad de los puntajes asignados. En el caso de la acreditación ¿con qué criterios eligen las unidades académicas a las agencias que las evaluarán? Para asegurar la neutralidad y comparabilidad de los procesos sería más aconsejable que la CNA asignara aleatoriamente a las agencias, para

10 Por ejemplo, la longitud de dichos acuerdos varía entre cuatro y ocho páginas, algunos se refieren a todos los criterios, otros no, algunos ni siquiera mencionan la carrera específica a la que se refiere la acreditación. 
lo cual, a su vez necesitaría contar con mecanismos y recursos que permitan asegurar la equivalencia entre ellas.

e) El financiamiento de la acreditación

Cómo y quién financia el proceso de acreditación es otro punto delicado del diseño del sistema, en tanto puede crear incentivos contradictorios para sus actores. También aquí se evidencia una "herencia" del sistema voluntario, si bien puede resultar comprensible que una institución deba pagar por un servicio de acreditación, esto resulta menos claro si debe someterse obligatoriamente al proceso. Al respecto, es posible encontrar en la experiencia internacional casos de procesos de acreditación que son libres de pago para las instituciones, y otros en que se cobra un arancel a la institución, como es el caso de NCATE ${ }^{11}$ en Estados Unidos (Ingvarson et al, 2006). Sin embargo, esta se trata de una acreditación voluntaria, donde, a diferencia del caso chileno, la agencia no tiene fines de lucro.

En Chile, los aranceles de la CNA para la acreditación de programas de pregrado son fijados por la Dirección de Presupuestos del Ministerio de Hacienda en pesos y definen un factor de ajuste según la distancia de las sedes que se deben evaluar. Los aranceles de las agencias son definidos e informados en UF por ellas mismas (tres de las cuatro, los tienen disponibles en su sitio web), pero el valor según el número de sedes a evaluar -sobre 2- no es informado.

Los aranceles de las agencias difieren aunque no exageradamente (oscilan entre 300 UF y 370 UF), no obstante, estas declaran disposición a negociar el monto, dependiendo del número de carreras afines y número de sedes a acreditar. El riesgo de dejar los aranceles definitivos en el terreno de la negociación entre agencia e institución es la contradicción de incentivos e intereses: serán precisamente las instituciones más precarias las que necesiten reducir los costos del proceso, empujando a las agencias a competir ofreciendo aranceles más convenientes, con el consiguiente impacto en la calidad del proceso. Lo que se cuestiona aquí no es la integridad de las agencias e instituciones, sino el diseño del sistema en que ellas operan -tal

11 National Council for the Accreditation of Teacher Education. 
180 ANÁLISIS Y PROPUESTAS PARA LA ACREDITACIÓN DE PEDAGOGÍAS EN CHILE - M. Domínguez, L. Meckes

como este quedó establecido en la Ley-y las contradicciones que por lo tanto pueden surgir.

Para disminuir las distorsiones de este sistema, algunos cambios que se puede sugerir son:

- Que las agencias acreditadoras no tengan una relación contractual directa con las instituciones de educación superior y que esta relación se establezca con la CNA o sea mediada por ella.

- Que los aranceles de las agencias sean idénticos (incluidas las variaciones de acuerdo a número de sedes a evaluar) y fijados centralmente del mismo modo que los de la CNA.

En síntesis, si la política educacional chilena quiere verdaderamente potenciar la acreditación de las pedagogías como herramienta para asegurar la preparación de quienes ingresarán a las aulas del país, necesita mejorar los criterios de evaluación, definiendo criterios específicos para los programas de formación inicial docente; elevar los niveles de exigencia de aquellos criterios con estándares de proceso y de resultados, y establecer consecuencias más severas para aquellas carreras que no se presentan al proceso o que resultan no acreditadas. Asimismo, el sistema requiere estandarizar los procedimientos, mecanismos y normas que siguen las agencias acreditadoras, y fortalecer la supervisión para garantizar la comparabilidad de los resultados entre distintas agencias, acorde a los requerimientos de una evaluación obligatoria y utilizada para fines de accountability.

\section{Referencias bibliográficas}

Ávalos, B. y Matus, C. (2010) La Formación Inicial Docente en Chile desde una Óptica Internacional. Informe Nacional del Estudio IEA TEDS-M. Santiago: Ministerio de Educación.

Barber, M. y Mourshed, M. (2008) Cómo hicieron los sistemas educativos con mejor desempeño para alcanzar sus objetivos. PREAL.

Bills, L.; Briggs, M.; Browne, A.; Gillespie, H.; Gordon, J.; Husbands, C.; Phillips, E.; Still, C. y Swatton, P. (2008) International perspectives on quality in initial teacher education: an exploratory review of selected 
international documentation on statutory requirements and quality assurance. En: Research Evidence in Education Library, London: EPPI-Centre, Social Science Research Unit, Institute of Education, University of London.

Comisión Nacional de Acreditación (2010) Aseguramiento de Calidad en la Educación Superior: Cuatro años de CNA-Chile. 2007-2010 Memoria. Santiago: CNA-Chile.

Comisión Nacional de Acreditación (2008) "Normas y procedimientos acreditación de pregrado".

Comisión sobre Formación Inicial de Docentes (2005) Informe Comisión sobre Formación Inicial Docente, Santiago: Ministerio de Educación y Organización de Estados Iberoamericanos para la Educación, la Ciencia y la Cultura (OEI).

Congreso Nacional de Chile (2009) Ley 20.129. Sistema Nacional de Aseguramiento de la Calidad de la Educación Superior.

Consejo Asesor Presidencial para la Calidad de la Educación (2006) Informe Final. Santiago.

Cox, C.; Meckes, L.; y Bascopé, M. (2011) La institucionalidad formadora de profesores en Chile en la década del 2000: velocidad del mercado y parsimonia de las políticas. Pensamiento Educativo (46-47), pp. 205245 .

Darling-H., L. y Bransford, J. (2005) Preparing Teachers for a Changing World. What should Teachers should learn and be able to do. San Francisco: Jossley Bass.

Darling-Hammond, L. (2006) Power Teacher Education. Lessons from Exemplary Programs. San Francisco: Jossley Bass.

Euridyce: Education, Audiovisual and Culture Executive Agency, (2010) Focus on Higher Education in Europe 2010: The Impact of the Bologna Process. Brussels: Euridyce.

Fuhrman, S. (2003) Rediseñando los Sistemas de Accountability para la Educación. Consortium for Policy Research in Education (CPRE). Policy Brief RB-38.

Ingvarson, L.; Elliott, A.; Kleinhenz, E. y McKenzie, P. (2006) Teacher education accreditation: a review of national and international trends and practices. ACER, Teaching Australia.

Ingvarson, L.; Schwille, J.; Rowley, G.; Tatto, M. T.; Senk, S. y Peck, R. (2011) National Policies and Regulatory Arrangements for the Mathematics Preparation of Future Teachers in Sixteen Countries. Amsterdam: 
182 ANÁLISIS Y PROPUESTAS PARA LA ACREDITACIÓN DE PEDAGOGÍAS EN CHILE - M. Domínguez, L. Meckes

International Association for the Evaluation of Educational Achievement (IEA), en prensa.

Lemaitre, M. J. y Zapata, G. (2003) Antecedentes, Situación Actual y Perspectivas de la Evaluación y la Acreditación de la Educación Superior en Chile. En: Políticas Públicas: Demandas Sociales y Gestión del Conocimiento. Santiago: Centro Interuniversitario de Desarrollo, CINDA Informe Nacional, Chile.

Levine, A. (2006) Educating School Teachers. Washington DC: The Education Schools Project.

National Council for Accreditation of Teacher Education (NCATE). The Standard of Excellence in Teacher Preparation (2008) Professional Standards for the Accreditation of Teacher Preparation Institutions. Washington: NCATE.

Navarro, Gabriela (2008) Impacto del proceso de acreditación de carreras en el mejoramiento de la gestión académica, Santiago: Consejo Superior de Educación.

OECD (2004) Revisión de políticas nacionales de educación: Chile. Santiago: OCDE.

OCDE (2005) Teachers matter: attracting, developing and retaining effective teachers, Paris: OCDE.

Panel de expertos para una educación de calidad (2010) Informe final. Propuestas para fortalecer la profesión docente en el sistema escolar Chileno. Santiago: Ministerio de Educación. Disponible en: http://www.mineduc.cl/biblio/ documento/201007091211380.Informe\%20final.pdf

Quality Assurance Agency, QAA (2007) Standards for Initial Teacher Training, Quality Assurance Agency, Scotland

Ravela, P.; Arregui, P.; Valverde, G.; Wolfe, R.; Ferrer G.; Martínez R., Felipe; Aylwin, M.; Wolff, L. (2008) Las evaluaciones educativas que América Latina necesita Documento n. ${ }^{\circ} 40$. Santiago: PREAL.

Rothstein, R.; Jacobsen, R. y Wilder, T. (2009) From Accreditation to Accountability. Phi Delta Kappan (90) pp. 624-630.

Scharager, J. y Aravena, T. (2010) Impacto de las políticas de aseguramiento de la calidad en programas de educación superior: un estudio exploratorio. Calidad en la Educación. Consejo Nacional de Educación, n. ${ }^{\circ}$ 32, pp. 15-42.

Sotomayor, C.; Parodi, G.; Coloma, C. J.; Ibáñez, R., y Cavada, P. (2010) La formación inicial de docentes de educación general básica en Chile: ¿Qué se espera que aprendan los futuros profesores en el área de Lenguaje y 
Comunicación? Centro de Investigación Avanzada en Educación (CIAE). Universidad de Chile - Pontificia Universidad Católica de Valparaíso.

Stensaker, B. (2007) Impact of quality processes. En: Bollaert et al. (Eds.), Embedding quality culture in higher education. Brussels: European University Association.

Teacher Education Accreditation Council, TEAC (2009) Guide to Accreditation, TEAC, Washington.

Disponible en: www.teac.org/wp-content/uploads/2009/03/teac-guideto-accreditation.pdf

Teacher Development Agency, TDA (2006). Handbook of guidance, that accompanies Qualifying to teach: Professional standards for qualified teacher status and requirements for initial teacher training, UK.

Teacher Development Agency, TDA (2008) Professional Standards for Qualified Teacher Status and Requirements for Initial Teacher Training, UK.

Varas, L.; Felmer, P.; Gálvez, G.; Lewin, R.; Martínez, C.; Navarro, S.; Ortiz, A., y Schwarze, G., (2008) Oportunidades de preparación para enseñar matemática de futuros profesores de educación general básica en Chile, Calidad de la Educación, № 29, Consejo Superior de Educación.

Wang, A. H.; Coleman, A. B.; Coley, R. J., y Phelps, R. P. (2003) Preparing Teachers around the World. Princeton: Educational Testing Service.

Wilson, S. y Youngs, P. (2006) Research on Accountability Processes in Teacher Education. En: Cochran-Smith y Zeichner (2005) Studying Teacher Education: The Report of the AERA Panel on Research and Teacher Education. Mahwah NJ: Lawrence Erlbaum Associates, Inc.

Zapata, G. y Tejeda, I. (2009) Informe Nacional Chile Educación Superior y Mecanismos de Aseguramiento de la Calidad. Proyecto ALFA "Aseguramiento de la Calidad: Políticas Públicas y Gestión Universitaria". Santiago: Centro Interuniversitario de Desarrollo, CINDA.

Recibido: 05/04/2011

Aceptado: 09/06/2011 